\title{
Space-Time Geometry of Electromagnetic Field in the System of Photon
}

\author{
Mukul Chandra Das ${ }^{1,2, *}$, Rampada Misra ${ }^{3}$ \\ ${ }^{1}$ Singhania University, Pacheri Bari - 333515, Jhunjhunu, Rajasthan, India \\ ${ }^{2}$ Satmile High School, Satmile - 721452, Contai, West Bengal, India \\ ${ }^{3}$ Department of Electronics, Vidyasagar University, Midnapore-721102, West Bengal, India \\ *E-mail address: mukuldas.100@gmail.com
}

\begin{abstract}
In the concept of general relativity gravity is the space-time geometry. Again, a relation between electromagnetic field and gravitational field is expected. In this paper, space-time geometry of electromagnetic field in the system of photon has been introduced to unify electromagnetic field and gravitational field in flat and curvature space-time.
\end{abstract}

Keywords: space-time geometry; unified field; electromagnetic field

\section{INTRODUCTION}

In physics, a unified field theory is a type that allows all fundamental forces and elementary particles to be written in terms of a single field.

The term was proposed by Einstein, who attempted to unify the general theory of relativity with electromagnetism. According to Einstein's general relativity [2,3], gravity is the space-time geometry. Also, he suggested [4] the field equation for the gravity of an electromagnetic wave as $G_{\alpha \beta}=-K T(E)_{a b}$ where, $G_{\alpha \beta}$ is the Einstein tensor, and $K$ is the coupling constant. But, the problem of the unification of fundamental fields into a single theory has not been solved until now in a satisfactory manner, although, in different time, a lot of papers have been published which attempt to unify the fundamental fields.

Recently, in [1], a relation between electromagnetic field and gravitational field has been introduced by considering a super system in photon. In this paper a trial has been made to introduce a geometrical relation between electromagnetic field and gravitational field.

\section{SPACE-TIME GEOMETRY OF SYSTEMS}

In [1], to clarify two simultaneous superimposed motion (either linear or rotational), three types of system has been assumed which are L-L system, S-S system and S-L system;

depending upon the S-L system SSP picture of photon has been considered; also, using this picture (SSP) a connection between electro-magnetic field $\left(\psi_{\alpha}(r, t)\right)$ and gravitational field $\left(G_{\alpha}^{\prime}\left(r^{\prime}, t^{\prime}\right)\right)$ has been introduced by the relation 


$$
\psi_{\alpha}(r, t)=\Upsilon_{1} \bar{Z}_{i j} G_{\alpha}^{\prime}\left(r^{\prime}, t^{\prime}\right)
$$

where, $\bar{Z}_{i j}$ are transformation matrix in the picture of SSP.

It is also pointed out that to clarify L-L or S-S or S-L system, four reference frames $\left(S, S_{1}, S_{2}\right.$, $S_{3}$ ) has been considered in a simultaneous superimposed form.

Relation for co-ordinate transformation from $S_{3}$ to $S$ in S-L system [1] is

$$
X(x, y, z, t)=\bar{Z}_{i j} X^{\prime}\left(x^{\prime}, y^{\prime}, z^{\prime}, t^{\prime}\right)
$$

where, $\bar{Z}_{i j}$ is co-ordinate transformation matrix and the co-ordinates of an event in $S_{3}$ be

$$
X^{\prime}\left(x^{\prime}, y^{\prime}, z^{\prime}, t^{\prime}\right)=\left(\begin{array}{c}
x^{\prime} \\
y^{\prime} \\
z^{\prime} \\
t^{\prime}
\end{array}\right) \text { which would be } X(x, y, z, t)=\left(\begin{array}{c}
x \\
y \\
z \\
t
\end{array}\right) \text { with respectively in } S .
$$

Now, following the space-time geometry as in [5], one can introduced the Space-time geometry of the said system as stated below

From (2) we obtain

$$
\begin{aligned}
& d x^{2}=\left(\bar{Z}_{11} d x^{\prime}+\bar{Z}_{12} d y^{\prime}+\bar{Z}_{13} d z^{\prime}+\bar{Z}_{14} d t^{\prime}\right)^{2} \\
& d y^{2}=\left(\bar{Z}_{21} d x^{\prime}+\bar{Z}_{22} d y^{\prime}+\bar{Z}_{23} d z^{\prime}+\bar{Z}_{24} d t^{\prime}\right)^{2} \\
& d z^{2}=\left(\bar{Z}_{31} d x^{\prime}+\bar{Z}_{32} d y^{\prime}+\bar{Z}_{33} d z^{\prime}+\bar{Z}_{34} d t^{\prime}\right)^{2} \\
& d t^{2}=\left(\bar{Z}_{41} d x^{\prime}+\bar{Z}_{42} d y^{\prime}+\bar{Z}_{43} d z^{\prime}+\bar{Z}_{44} d t^{\prime}\right)^{2}
\end{aligned}
$$

Now, we have Cartesian Co-ordinate geometry in flat space-time

$$
d s^{2}=-d t^{2}+d x^{2}+d y^{2}+d z^{2}
$$

Using (3) we obtain from (4) the space-time geometry in S-L system

$$
\begin{array}{r}
d s^{2}=P_{1} d x^{\prime 2}+P_{2} d y^{\prime 2}+P_{3} d z^{\prime 2}+P_{4} d t^{\prime 2}+2\left(\mathrm{Q}_{1} d x^{\prime} d y^{\prime}+\mathrm{Q}_{2} d x^{\prime} d z^{\prime}\right. \\
\left.+\mathrm{Q}_{3} d x^{\prime} d t^{\prime}+\mathrm{Q}_{4} d y^{\prime} d z^{\prime}+\mathrm{Q}_{5} d y^{\prime} d t^{\prime}+\mathrm{Q}_{6} d z^{\prime} d t^{\prime}\right)
\end{array}
$$

where,

$$
P_{1}=\bar{Z}_{11}{ }^{2}+\bar{Z}_{21}{ }^{2}+\bar{Z}_{31}{ }^{2}-\bar{Z}_{41}{ }^{2}, \quad P_{2}=\bar{Z}_{12}{ }^{2}+\bar{Z}_{22}{ }^{2}+\bar{Z}_{32}{ }^{2}-\bar{Z}_{42}{ }^{2}
$$




$$
\begin{array}{cc}
P_{3}=\bar{Z}_{13}{ }^{2}+\bar{Z}_{23}{ }^{2}+\bar{Z}_{33}{ }^{2}-\bar{Z}_{43}{ }^{2}, & P_{4}=\bar{Z}_{14}{ }^{2}+\bar{Z}_{24}{ }^{2}+\bar{Z}_{34}{ }^{2}-\bar{Z}_{44}{ }^{2} \\
\mathrm{Q}_{1}=\bar{Z}_{11} \bar{Z}_{12}+\bar{Z}_{21} \bar{Z}_{22}+\bar{Z}_{31} \bar{Z}_{32}-\bar{Z}_{41} \bar{Z}_{42}, & \mathrm{Q}_{2}=\bar{Z}_{11} \bar{Z}_{13}+\bar{Z}_{21} \bar{Z}_{23}+\bar{Z}_{31} \bar{Z}_{33}-\bar{Z}_{41} \bar{Z}_{43} \\
\mathrm{Q}_{3}=\bar{Z}_{11} \bar{Z}_{14}+\bar{Z}_{21} \bar{Z}_{24}+\bar{Z}_{31} \bar{Z}_{34}-\bar{Z}_{41} \bar{Z}_{44}, & \mathrm{Q}_{4}=\bar{Z}_{12} \bar{Z}_{13}+\bar{Z}_{22} \bar{Z}_{23}+\bar{Z}_{32} \bar{Z}_{33}-\bar{Z}_{42} \bar{Z}_{43} \\
\mathrm{Q}_{5}=\bar{Z}_{12} \bar{Z}_{14}+\bar{Z}_{22} \bar{Z}_{24}+\bar{Z}_{32} \bar{Z}_{34}-\bar{Z}_{42} \bar{Z}_{44}, & \mathrm{Q}_{6}=\bar{Z}_{13} \bar{Z}_{14}+\bar{Z}_{23} \bar{Z}_{24}+\bar{Z}_{33} \bar{Z}_{34}-\bar{Z}_{43} \bar{Z}_{44}
\end{array}
$$

Again, relation for co-ordinate transformation from $S_{3}$ to $S$ in S-S system [1] is

$$
X(x, y, z, t)=\bar{S}_{i j} X^{\prime}\left(x^{\prime}, y^{\prime}, z^{\prime}, t^{\prime}\right)
$$

where, $\bar{S}_{i j}$ is co-ordinate transformation matrix.

From (6) we obtain

$$
\begin{aligned}
& d x^{2}=\left(\bar{S}_{11} d x^{\prime}+\bar{S}_{12} d y^{\prime}+\bar{S}_{13} d z^{\prime}+\bar{S}_{14} d t^{\prime}\right)^{2} \\
& d y^{2}=\left(\bar{S}_{21} d x^{\prime}+\bar{S}_{22} d y^{\prime}+\bar{S}_{23} d z^{\prime}+\bar{S}_{24} d t^{\prime}\right)^{2} \\
& d z^{2}=\left(\bar{S}_{31} d x^{\prime}+\bar{S}_{32} d y^{\prime}+\bar{S}_{33} d z^{\prime}+\bar{S}_{34} d t^{\prime}\right)^{2} \\
& d t^{2}=\left(\bar{S}_{41} d x^{\prime}+\bar{S}_{42} d y^{\prime}+\bar{S}_{43} d z^{\prime}+\bar{S}_{44} d t^{\prime}\right)^{2}
\end{aligned}
$$

Using (4) and (7) we obtain the space-time geometry in S-S system as in (5) where,

$$
\begin{array}{cc}
P_{1}=\bar{S}_{11}{ }^{2}+\bar{S}_{21}{ }^{2}+\bar{S}_{31}{ }^{2}-\bar{S}_{41}{ }^{2}, & P_{2}=\bar{S}_{12}{ }^{2}+\bar{S}_{22}{ }^{2}+\bar{S}_{32}{ }^{2}-\bar{S}_{42}{ }^{2} \\
P_{3}=\bar{S}_{13}{ }^{2}+\bar{S}_{23}{ }^{2}+\bar{S}_{33}{ }^{2}-\bar{S}_{43}{ }^{2}, & P_{4}=\bar{S}_{14}{ }^{2}+\bar{S}_{24}{ }^{2}+\bar{S}_{34}{ }^{2}-\bar{S}_{44}{ }^{2} \\
\mathrm{Q}_{1}=\bar{S}_{11} \bar{S}_{12}+\bar{S}_{21} \bar{S}_{22}+\bar{S}_{31} \bar{S}_{32}-\bar{S}_{41} \bar{S}_{42}, & \mathrm{Q}_{2}=\bar{S}_{11} \bar{S}_{13}+\bar{S}_{21} \bar{S}_{23}+\bar{S}_{31} \bar{S}_{33}-\bar{S}_{41} \bar{S}_{43} \\
\mathrm{Q}_{3}=\bar{S}_{11} \bar{S}_{14}+\bar{S}_{21} \bar{S}_{24}+\bar{S}_{31} \bar{S}_{34}-\bar{S}_{41} \bar{S}_{44}, & \mathrm{Q}_{4}=\bar{S}_{12} \bar{S}_{13}+\bar{S}_{22} \bar{S}_{23}+\bar{S}_{32} \bar{S}_{33}-\bar{S}_{42} \bar{S}_{43} \\
\mathrm{Q}_{5}=\bar{S}_{12} \bar{S}_{14}+\bar{S}_{22} \bar{S}_{24}+\bar{S}_{32} \bar{S}_{34}-\bar{S}_{42} \bar{S}_{44}, & \mathrm{Q}_{6}=\bar{S}_{13} \bar{S}_{14}+\bar{S}_{23} \bar{S}_{24}+\bar{S}_{33} \bar{S}_{34}-\bar{S}_{43} \bar{S}_{44}
\end{array}
$$

\section{SPACE-TIME GEOMETRY OF ELECTROMAGNETIC FIELD IN PHOTON}

Since picture of SSP depends upon the S-L system so, following (1) and using $d x^{\prime}=d x^{g}, d y^{\prime}=d y^{g}, d z^{\prime}=d z^{g}, d t^{\prime}=d t^{g}$ we obtain from (5), the space-time geometry of electromagnetic field in the SSP

$$
\begin{array}{r}
\left(d s^{e m}\right)^{2}=P_{1}\left(d x^{g}\right)^{2}+P_{2}\left(d y^{g}\right)^{2}+P_{3}\left(d z^{g}\right)^{2}+P_{4}\left(d t^{g}\right)^{2}+2\left(\mathrm{Q}_{1} d x^{g} d y^{g}\right. \\
\left.+\mathrm{Q}_{2} d x^{g} d z^{g}+\mathrm{Q}_{3} d x^{g} d t^{g}+\mathrm{Q}_{4} d y^{g} d z^{g}+\mathrm{Q}_{5} d y^{g} d t^{g}+\mathrm{Q}_{6} d z^{g} d t^{g}\right)
\end{array}
$$

where, superscript ' $\mathrm{g}$ ' represents the gravitational system and superscript em represents electromagnetic system. 
Following the convention as in (1), one may assume a relation between electromagnetic field and gravitational field as

$$
\psi_{\alpha}(r, t)=\bar{\Upsilon}_{1} \bar{S}_{i j} G_{\alpha}^{\prime}\left(r^{\prime}, t^{\prime}\right)
$$

where, $\bar{\Upsilon}_{1}$ is a constant and $\bar{S}_{i j}$ is transformation matrix in S-S system.

This means that, in S-S system, gravitational field of frame $S_{3}$ would be electromagnetic field with respect to frame $S$. For this system space-time geometry of electromagnetic field would also be as in (8) where, transformation matrix would be $\bar{S}_{i j}$

However, equation (8) would be the space-time geometry of electromagnetic field connecting gravitational field and electromagnetic field in the system of photon.

\section{CONCLUSION}

Equation (8) represents a picture of space-time geometry of the electromagnetic field in the system of photon. This implies that a geometrical relation is existed in between electromagnetic field and gravitational field.

\section{References}

[1] M. C. Das, R. Misra, International Letters of Chemistry, Physics and Astronomy 7(2) (2013) 73-84.

[2] J. B. Hartle, “Gravity: An Introduction to Einstein's General Relativity,"3rd Edition, Pearson Education Inc, India, 2009,

[3] A. Einstein, Annalen der Physik 354(7) (1916) 769-822, doi:10.1002/andp.19163540702.

[4] Einstein A., Physics and relativity (1936) in Ideas and Opinions, Crown, New York 1954, p. 311.

[5] M. C. Das, R. Misra, International Letters of Chemistry, Physics and Astronomy 8(3) (2013) 215-219. 